\title{
New Advances in Kotzig's Conjecture
}

\author{
Christian Barrientos $^{1^{*}}$ and Sarah Minion ${ }^{2}$ \\ ${ }^{1}$ Department of Mathematics, East Campus, Valencia College, Orlando, Florida, U.S.A. \\ ${ }^{2}$ Department of Mathematics, Full Sail University, Orlando, Florida, U.S.A. \\ *Corresponding author E-mail: chr_barrientos@yahoo.com
}

\begin{abstract}
Article Info
Keywords: Decomposition, Graceful graph, Graceful labeling, $\rho$-labeling 2010 AMS: 05C51, $05 C 78$

Received: 13 May 2019

Accepted: 29 August 2019

Available online: 20 December 2019
\end{abstract}

\begin{abstract}
In 1973 Kotzig conjectures that the complete graph $K_{2 n+1}$ can be cyclically decomposed into $2 n+1$ copies of any tree of size $n$. Rosa proved that this decomposition exists if and only if there exists a $\rho$-labeling of the tree. In this work we prove that if $T^{\prime}$ is a graceful tree, then any tree $T$ obtained from $T^{\prime}$ by attaching a total of $k \geq 1$ pendant vertices to any collection of $r$ vertices of $T^{\prime}$, where $1 \leq r \leq k$, admits a $\rho$-labeling. As a consequence of this result, many new families of trees with this kind of labeling are produced, which indicates the strong potential of this result. Moreover, the technique used to prove this result, gives us an indication of how to determine whether a given tree of size $n$ decomposes the complete graph $K_{2 n+1}$. We also prove the existence of a $\rho$-labeling for two subfamilies of lobsters and present a method to produce $\rho$-labeled trees attaching pendant vertices and pendant copies of the path $P_{3}$ to some of the vertices of any graceful tree.

In addition, for any given tree $T$, we use bipartite labelings to show that this tree is a spanning tree of a graph $G$ that admits an $\alpha$-labeling. This is not a new result; however, the construction presented here optimizes (reduces) the size of $G$ with respect to all the similar results that we found in the literature.
\end{abstract}

\section{Introduction}

A decomposition (or edge-decomposition) of the complete graph $K_{n}$ is a system $R$ of subgraphs such that any edge of $K_{n}$ belongs to exactly one of the subgraphs in $R$. Suppose that the vertices of $K_{n}$ are labeled $0,1, \ldots, n-1$; let $i j \in E\left(K_{n}\right)$, a turning of the edge $i j$ is the increase of both labels by one, i.e., the edge $(i+1)(j+1)$, where the addition is taken modulo $n$. A turning of a subgraph $G$ of $K_{n}$ is the simultaneous turning of all the edges of $G$. A decomposition $R$ of $K_{n}$ is called cyclic when for every $G$ in $R$, the turning $G^{\prime}$ of $G$ is also in $R$.

In 1963, Ringel [1] presented the following conjecture: If $T$ is a tree of size $n$, then the complete graph $K_{2 n+1}$ is edge-decomposable into $2 n+1$ copies of $T$. Ten years later, Kotzig [2] stated the following variation of this conjecture: The complete graph $K_{2 n+1}$ can be cyclically decomposed into $2 n+1$ subgraphs isomorphic to a given tree with $n$ edges. In 1966, Rosa [3] introduced four valuations (or labelings) of the vertices of a graph that can be used to find a cyclic decomposition of $K_{2 n+1}$. A difference vertex labeling of a graph $G$ of size $n$ is an injective mapping $f: V(G) \rightarrow S$, where $S$ is a set of nonnegative integers, such that every edge $u v$ of $G$ has assigned a weight defined by $|f(v)-f(u)|$. All labelings considered in this work are difference vertex labelings. Rosa's valuations can be described as follows.

Suppose that $f$ is a labeling of a graph $G$ of size $n$. Let $L_{f}$ be the set of labels assigned by $f$ to the vertices of $G$ and $W_{f}$ be the set of weights induced by $f$ on the edges of $G$. Consider the following conditions.

(a) $L_{f} \subseteq\{0,1, \ldots, n\}$

(b) $L_{f} \subseteq\{0,1, \ldots, 2 n\}$

(c) $W_{f}=\{1,2, \ldots, n\}$

(d) $W_{f}=\left\{w_{1}, w_{2}, \ldots, w_{n}\right\}$ where $w_{i}=i$ or $w_{i}=2 n+1-i$ for every $1 \leq i \leq n$

(e) it exists $\lambda$ in $\{0,1, \ldots, n\}$, such that for any arbitrary edge $u v$ of $G$, either $f(u) \leq \lambda<f(v)$ or $f(v) \leq \lambda<f(u)$. (The number $\lambda$ is called the boundary value of $f$.)

Email addresses and ORCID numbers: chr_barrientos@yahoo.com, https://orcid.org/0000-0003-2838-8687 (C. Barrientos), sarah.m.minion@gmail.com, https://orcid.org/0000-0002-8523-3369 (S. Minion) 


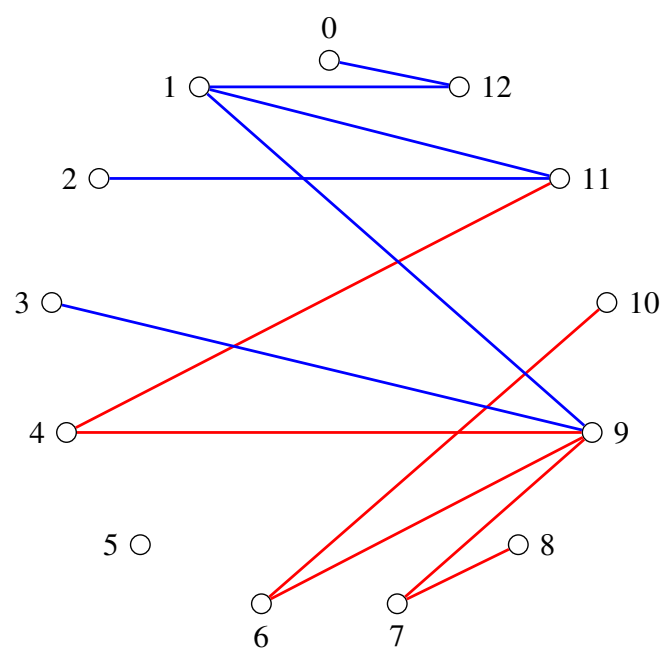

Figure 1.1: A $\rho$-labeling of $S(3,2)$ and its 8th turning

\begin{tabular}{|c|c|c|c|c|c|c|c|c|c|c|c|c|}
\hline+0 & +1 & +2 & +3 & +4 & +5 & +6 & +7 & +8 & +9 & +10 & +11 & +12 \\
\hline $0-12$ & 1-0 & $2-1$ & $3-2$ & $4-3$ & $5-4$ & $6-5$ & $7-6$ & $8-7$ & $9-8$ & $10-9$ & $11-10$ & $12-11$ \\
\hline $1-12$ & $2-0$ & $3-1$ & $4-2$ & $5-3$ & $6-4$ & $7-5$ & $8-6$ & $9-7$ & $10-8$ & $11-9$ & $12-10$ & $0-11$ \\
\hline $2-11$ & $3-12$ & $4-0$ & $5-1$ & $6-2$ & $7-3$ & $8-4$ & $9-5$ & $10-6$ & $11-7$ & $12-8$ & $0-9$ & $1-10$ \\
\hline $1-9$ & $2-10$ & $3-11$ & $4-12$ & $5-0$ & $6-1$ & $7-2$ & $8-3$ & $9-4$ & $10-5$ & $11-6$ & $12-7$ & $0-8$ \\
\hline $3-9$ & $4-10$ & $5-11$ & $6-12$ & $7-0$ & $8-1$ & $9-2$ & $10-3$ & $11-4$ & $12-5$ & $0-6$ & $1-7$ & $2-8$ \\
\hline
\end{tabular}

Table 1: $\rho$-labeling of $S(3,2)$ and all its turnings

When $f$ satisfies the conditions (a), (c), and (e), it is called an $\alpha$-labeling (and $G$ is designated an $\alpha$-graph). If $f$ satisfies the conditions (a) and (c), then it is called a $\beta$-labeling or graceful labeling (and $G$ is named a graceful graph). The function $f$ is a $\sigma$-labeling if (b) and (c) hold (in this case $G$ is a $\sigma$-graph). A $\rho$-labeling must satisfies the conditions (b) and (d); in this case, $G$ is named a $\rho$-graph. Thus, every $\alpha$-graph is a graceful graph, every graceful graph is a $\sigma$-graph, and every $\sigma$-graph is a $\rho$-graph. Using these labelings, Rosa [3] proved the following theorem.

Theorem 1.1. A cyclic decomposition of the complete graph $K_{2 n+1}$ into subgraphs isomorphic to a given graph $G$ of size $n$ exists if and only if there exists a $\rho$-labeling of $G$.

Consequently, Kotzig's conjecture can be stated in terms of $\rho$-labelings as follows.

Conjecture 1. Every tree of size $n$ is a $\rho$-tree.

The tree $S(3,2)$, obtained by attaching a pendant vertex to every leaf of the star $S_{3} \cong K_{1,3}$, is the smallest tree that is not an $\alpha$-tree. Suppose that the vertices of $K_{1,3}$ are labeled $0,1, \ldots, 12$. Thus, every column in Table 1 shows the adjacencies of the vertices of $S(3,2)$ within $K_{1,3}$, being the first column a $\rho$-labeling of this tree and every column after that corresponds to a turning of the previous labeled graph. In Figure 1.1 we show, in blue, the $\rho$-labeling of $S(3,2)$ used to create the cyclic decomposition of $K_{1,3}$, together with its 8 th turning, represented in red.

In Section 2 we show that a tree $T$ admists a $\rho$-labeling if it has a graceful subtree $T^{\prime}$, such that $T^{\prime}$ can be obtained by deleting a number of leaves of $T$. Given that several families of graceful trees are known, this result allows us to expand, considerably, the number of trees or families of trees that admit a $\rho$-labeling, therefore, decompose the complete graph $K_{2 n+1}$. Also here, we show two subfamilies of 3-distance trees that admit $\rho$-labelings. In addition, we study the existence of $\rho$-labelings for trees obtained from smaller graceful trees by attaching copies of the path $P_{3}$ to some selected vertices of the base graceful trees; these selected vertices may be chosen almost randomly.

In [4], Barrientos and Krop represented a tree as an ordered rooted tree to calculate its excess $\varepsilon(T)$. This parameter was used in [5] to find a $\rho$-labeling for any tree $T$ that containing a branch that is a caterpillar of size at least $\varepsilon(T)$. In Section 3, we use the parameter $\varepsilon(T)$ to show the existence of an $\alpha$-graph of size $n+\varepsilon(T)$ that contains $T$ as a spanning tree.

All graphs used in this paper are finite, with no loops nor multiple edges. We follow the notation and terminology used in [6] and [7].

\section{Constructing $\rho$-graphs from graceful graphs}

Several families of $\rho$-trees are known. Gallian [7] mentions that in an unpublished work of Caro et al., [8], it was proven that all graphs with at most 11 edges have a $\rho$-labeling as well as lobsters. Késdy [9] defined a stunted tree as follows: a tree of size $n$ is stunted if its edges can be linearly ordered $e_{1}, e_{2}, \ldots, e_{n}$ so that $e_{1}$ and $e_{2}$ share a vertex and, for all $3 \leq j \leq n, e_{j}$ shares a vertex with at least one $e_{k}$ such that $2 k \leq j-1$. He proved that if $p=2 n+1$ is prime, then every stunted tree of size $n$ has a $\rho$-labeling.

A spider is a tree that has at most one vertex, called the center, of degree greater than 2. Bahls et al. [10], proved that spiders for which the lengths of every path from the center to a leaf differ by at most one, are graceful. A comet is a spider where all the paths used have equal 
length. El-Zanati et al. [11], proved that trees of diameter at most 5, lobsters, and comets admit a more restrictive type of $\rho$-labeling, called $\rho^{+}$-labeling. Essentially, $\rho^{+}$-labelings are $\rho$-labelings with the extra condition of being bipartite (see Section 3 for a formal definition of bipartite labeling). This extra condition is what it makes their result a novelty.

\subsection{The first expansion}

Let $T$ be a tree of positive size $q$ with at least $k$ leaves and $T^{\prime}$ be any tree of size $q-k$ obtained from $T$ by deleting $k$ of its leaves. We claim that $T$ admits a $\rho$-labeling when $T^{\prime}$ is a graceful tree.

Theorem 2.1. Let $T^{\prime}$ be any tree obtained from $T$ by deleting any $k$ of its leaves. If $T^{\prime}$ is graceful, then $T$ admits a $\rho$-labeling.

Proof. Let $T$ be a tree of size $q$ with at least $k$ leaves and $T^{\prime}$ be a graceful tree obtained by deleting $k$ leaves from $T$. Suppose that $f$ is a graceful labeling of $T^{\prime}$. Thus, the labels assigned by $f$ to the vertices of $T^{\prime}$ are $0,1, \ldots, q-k$ and the weights induced by $f$ on the edges of $T^{\prime}$ are $1,2, \ldots, q-k$. Let $v_{1}, v_{2}, \ldots, v_{r}$ be the vertices of $T^{\prime}$ that when seen as vertices of $T$ corresponds to those incident to the edges of $T$ that were deleted to form $T^{\prime}$. Without loss of generality we assume that $f\left(v_{1}\right)>f\left(v_{2}\right)>\cdots>f\left(v_{r}\right)$. Thus, $f\left(v_{1}\right) \leq q-k$ and $f\left(v_{r}\right) \geq 0$.

In any $\rho$-labeling of a graph of size $q$, the labels are taken from $\{0,1, \ldots, 2 q\}$. When the labeled $T^{\prime}$ is seen inside $T$, its labeling can be extended to a $\rho$-labeling of $T$ as follows. There are $k$ leaves of $T$ that have not been labeled yet; none of the integers in $\{q-k+1, q-k+2, \ldots, 2 q\}$ have been used as a label; none of the integers $q-k+1, q-k+2, \ldots, q$ correspond to the weight of an edge of $T$. Since we are constructing a $\rho$-labeling of $T$, instead of these weights we use their complements with respect to $2 q+1$, that is, $q+k, q+k-1, \ldots, q+1$, respectively. For every $i \in\{1,2, \ldots, r\}$, let $s_{i}$ be the number of edges incident to $v_{i}$ that were deleted from $T$ to form $T^{\prime}$. Starting with $v_{1}$, these edges are going to have, the still not assigned, $s_{i}$ largest weights, in the list $q+k, q+k-1, \ldots, q+1$, and so on. In order to see that this is possible, note that the label $q+k+f\left(v_{1}\right) \leq q+k+q-k \leq 2 q$ can be assigned to a leaf adjacent to $v_{r}$. This means that given the graceful labeling of $T^{\prime}$ and the set of integers that have not been used as labels, we can produce edges whose weights are $q+k, q+k-1, \ldots, q+1$.

All the pendant unlabeled vertices attached to $v_{i}$, for every $1 \leq i \leq r$, are labeled with consecutive integers, inducing consecutive weights. If $m$ is the smallest label assigned to a leaf adjacent to $v_{i}$, inducing the weight $w=q+t \in\{q+1, q+2, \ldots, q+k\}$ for some $1 \leq t<k$, then the largest label assigned to a leaf adjacent to $v_{i+1}$ is at most $m-2$. In fact, given that $m-f\left(v_{i}\right)=q+t$, equivalently, $m=f\left(v_{i}\right)+q+t$, when $x$ is the largest label used on a leaf adjacent to $v_{i+1}$, then

$$
\begin{array}{r}
x-f\left(v_{i+1}\right)=q+t-1 \\
x=f\left(v_{i+1}\right)+q+t-1 \\
x<\left(f\left(v_{i}\right)+q+t\right)-1 \\
x<m-1 \\
x \leq m-2 .
\end{array}
$$

Hence, there is always an integer in $\{q+1, q+2, \ldots, 2 q\}$ that can be assigned as the label of a leaf, attached to $v_{i}$, to produce the required weight for that edge. Therefore, we have obtained a $\rho$-labeling of $T$.

In the rest of this section we show some results that can be obtained as a direct consequence of Theorem 2.1.

By definition, a lobster is a tree such that the deletion of all its pendant edges results in a caterpillar and caterpillars are graceful [3], therefore, using Theorem 2.1 we may prove the following corollary.

Corollary 2.2. All lobsters admit $\rho$-labelings.

In Figure 2.1 we show an example of this labeling for a lobster $L$ of size 38. The edges of $L$ represented by red lines are the ones that we deleted to obtain a caterpillar. As we mentioned at the beginning of this section, the fact that lobsters are $\rho$-trees has been proven in [8] and [11]; however, the earliest proof of it was given by Huang and Rosa in 1978 [12].

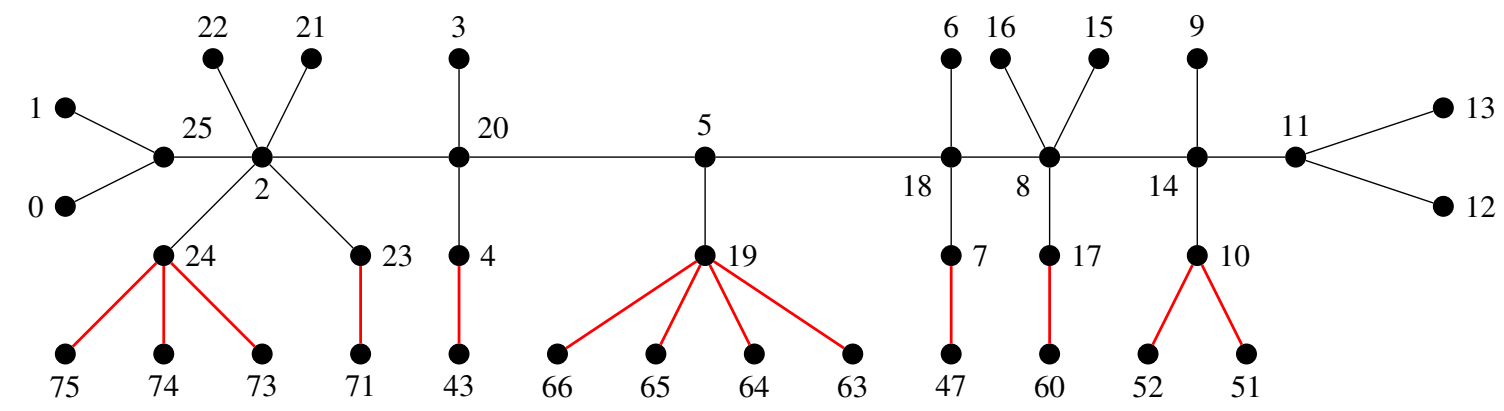

Figure 2.1: $\rho$-labeling of a lobster of size 38

In the same line, we have the following two results. Morgan [13] introduced the concept of $k$-distance tree as follows. Let $P$ be any of the longest paths in a tree $T ; T$ is a $k$-distance tree if every vertex is at distance at most $k$ from $P$. We refer to $P$ as the central path of $T$. From 
this definition we have that paths, caterpillars, and lobsters are 0-, 1-, and 2-distance trees, respectively. Recall that a subdivision of a graph is a graph obtained from it by replacing edges with pairwise internally-disjoint paths. In [14], Burzio and Ferrarese have shown that the tree obtained from any graceful tree, by subdividing every edge the same number of times, is also graceful. We use this result to prove the existence of a $\rho$-labeling for the members of two subfamilies of 3-distance trees.

Let $T$ be a 3-distance tree. The symbols $T^{*}$ and $P^{*}$ are used here to designate the lobster obtained from $T$ by deleting all its leaves and the central path of $T^{*}$, respectively.

Theorem 2.3. Let $T$ be a 3-distance tree. If for every pair of vertices in $P^{*}$ of degree at least 3 , the distance between them is even, then $T$ is a $\rho$-tree.

Proof. Let assume that $T$ is a 3-distance tree such that for every pair of vertices $u, v$ in $P^{*}$, of degree at least 3, dist $(u, v)$ is even. Let $X$ be the subset of $V\left(T^{*}\right)$ that contains all the leaves at distance 1 from $P^{*}$. Then for every $x \in X$, there exists $y_{x} \in V\left(T^{*}\right)$ such that $x y_{x} \in E(T)$. Suppose that for every $x \in X$, the edge $x y_{x}$ has been added to $E\left(T^{*}\right)$; thus, each vertex $y_{x}$ is at distance 2 from $P^{*}$, which implies that the new tree, denoted by $L$, is a lobster where all the leaves not in $P^{*}$ are at distance 2 from $P^{*}$ and the vertices of degree at least 3 in $P^{*}$ are separated by an even number of edges. Then, the lobster $L$ can be obtained by subdividing once every edge of a caterpillar. Using the result in [14], we know that $L$ is a graceful tree. Since $T$ can be obtained from $L$ by attaching some pendant vertices, a $\rho$-labeling of $T$ can be constructed applying Theorem 2.1 to a gracefully labeled copy of $L$.

Similarly, but as a direct consequence of Theorem 2.1, we can prove that when $T$ is a 3-distance tree where every leaf at distance 2 from the central path is at distance 3 from a leaf and for every pair of vertices of degree at least 3 in $P^{*}$ the distance between them is a positive multiple of 3 , then $T$ admits a $\rho$-labeling. We just need to observe that the underneath $T$ is created by subdividing twice every edge of a caterpillar. So, this graph is graceful and $T$ is a $\rho$-graph by Theorem 2.1

Corollary 2.4. Let $T$ be a 3-distance tree. If for every pair of vertices in $P^{*}$ of degree at least 3, the distance between them is a positive multiple of 3 , and every leaf at distance 2 from $P^{*}$ is at distance 3 from a leaf, then $T$ is a $\rho$-tree.

Using the help of a computer, Fang [15] has shown that all trees of size up to 34 are graceful, therefore, they admit a $\rho$-labeling. This fact helps us to prove the following corollary of Theorem 2.1 .

Corollary 2.5. Let $T$ be a tree of size $q$ with at least $k$ leaves. Any tree $T^{\prime}$ of size $q-k$, obtained by deleting $k$ leaves from $T$, admits a $\rho$-labeling if $q-k \leq 34$.

In [16], Eshghi and Azimi presented an algorithm to find graceful labelings of larger graphs. They verified this method with all trees with 30 , 35 , and 40 vertices. Hence, the result in Corollary 2.3 is also valid when $q-k=39$.

It is well-known that all trees with at most four leaves are graceful [17], [18], and [19]. Based on this result we have the following corollary of Theorem 2.1 .

Corollary 2.6. If $T$ is a tree such that the removal of all its leaves results in a tree with at most four leaves, then $T$ admists a $\rho$-labeling.

It is also known that trees with diameter at most 5 are graceful [18], [20]. If $T$ is a tree of diameter 5 , it can be represented as a rooted tree where the root is any of the central vertices; thus, only one of the branches coming out of the root has height 3 . Suppose now that $A$ is a tree of diameter 6 . When $A$ is seen as a rooted tree, where the root is its central vertex, more than one of the branches coming out of the root has height 3, if for all, except one of these branches, the leaves in level 3 are deleted, a tree $T$ of diameter 5 is obtained, which is graceful. Therefore, we can obtain a $\rho$-labeling of $T$ applying the procedure described in Theorem 2.1. Furthermore, the same idea can be used to construct a $\rho$-labeling of any tree $A^{*}$ of diameter 7 , in this case, we delete all the leaves in levels 3 and 4 to obtain a tree of diameter 5 ; hence we can apply Theorem 2.1. Thus, we have proven the following corollary.

Corollary 2.7. If $T$ is a tree of diameter at most 7 , then $T$ admits a $\rho$-labeling.

There are several families of graceful rooted trees, for example, Bermond and Sotteau [21] proved that every rooted tree, in which every level contains vertices of the same degree, is graceful. A tree satisfying this condition is called symmetric. This result allows us to prove the following corollary.

Corollary 2.8. If $T$ is a rooted tree with the property that the removal of some of its leaves results in a symmetric tree, then $T$ admits a $\rho$-labeling.

In [22], Balbuena et al., proved that all trees having an even or quasi even degree sequence are graceful. They obtained a graceful labeling for this type of graphs by representing them as rooted trees. They worked with rooted trees of diameter $D$, where the root is the central vertex and has odd degree, and all the interior vertices have even degree (even degree sequence). The case of a quasi even degree sequence is similar, except that the vertices in the penultimate level have odd degree.

Corollary 2.9. If $T$ is a tree with the property that the removal of some of its leaves results in a tree with an even or quasi even degree sequence, then $T$ admist a $\rho$-labeling.

Sethuraman and Jesintha [23] proved that all rooted trees, in which every level contains leaves and the degrees of the internal vertices in the same level are equal, are graceful.

Corollary 2.10. If $T$ is a rooted tree with the property that the removal of some of its leaves results in a rooted tree where every level contains leaves and the internal vertices, in that level, have the same degree, then $T$ admits a $\rho$-labeling. 


\subsection{The second expansion}

We open this part exploring the existence of a $\rho$-labeling of a tree $T^{\prime}$ of size $n+2 k$ obtained, from a graceful tree $T$ of size $n$, identifying an end-vertex of a copy of the path $P_{3}$ to each of $k$ selected vertices of $T$.

\section{The Labeling Scheme}

Suppose that $f$ is a graceful labeling of a tree $T$ of size $n$. Thus, the labels assigned by $f$ to the vertices of $T$ are the integers $0,1, \ldots, n$ and the induced weights are $1,2, \ldots, n$. This labeling will be extended to a $\rho$-labeling of a tree of size $n+2 k$.

Let $v_{1}, v_{2}, \ldots, v_{k}$ be distinct vertices of $T$. We assume that $f\left(v_{1}\right)>f\left(v_{2}\right), \ldots,>f\left(v_{k}\right)$. For every $1 \leq i \leq k$, we say that the vertex set of the $i$ th copy of $P_{3}$ is $\left\{v_{i}, u_{i}, w_{i}\right\}$ and the edge set is $\left\{v_{i} u_{i}, u_{i} w_{i}\right\}$. Then the labeling $f$ of $T$ is extended to include the vertices $u_{i}$ and $w_{i}$, in the following way:

$$
f\left(u_{i}\right)=f\left(v_{i}\right)+n+4 k+1-i
$$

and

$$
f\left(w_{i}\right)=f\left(v_{i}\right)+3 k-2 i+1 .
$$

We claim that $f$ is a $\rho$-labeling of $T^{\prime}$ when $f\left(v_{k}\right) \geq n-k$.

Theorem 2.11. Let $f$ be a graceful labeling of a tree $T$ of size $n$ and $v_{1}, v_{2}, \ldots, v_{k}$ be distinct vertices of $T$. If $\min \left\{f\left(v_{i}\right): 1 \leq i \leq k\right\} \geq n-k$, then the tree $T^{\prime}$, obtained attaching an end-vertex of $P_{3}$ to each $v_{i}$, is a $\rho$-tree.

Proof. Suppose that the graceful labeling $f$ of $T$ has been extended to all the vertices of $T^{\prime}$ using the labeling scheme presented above. First, we verify that the weights induced by $f$ satisfy the conditions required by a $\rho$-labeling.

The edge $v_{i} u_{i}$ has weight

$$
f\left(u_{i}\right)-f\left(v_{i}\right)=\left(f\left(v_{i}\right)+n+4 k+1-i\right)-f\left(v_{i}\right)=n+4 k+1-i .
$$

Since $1 \leq i \leq k$, these weights form the set $\{n+4 k, n+4 k-1, \ldots, n+3 k+1\}$. But the complement of each of these numbers is $(2 n+4 k+1)-(n+4 k+1-i)=n+i$, which implies these weights are equivalent to the integers in $\{n+1, n+2, \ldots, n+k\}$.

The edge $u_{i} w_{i}$ has weight

$$
f\left(u_{i}\right)-f\left(w_{i}\right)=\left(f\left(v_{i}\right)+n+4 k+1-i\right)-\left(f\left(v_{i}\right)+3 k-2 i+1\right)=n+k+i .
$$

Then, these weights form the set $\{n+k+1, n+k+2, \ldots, n+2 k\}$.

Since the weights induced on the edges of $T$ are $1,2, \ldots, n$, the weights induced by $f$ on the edges of $T^{\prime}$ satisfy the conditions required by a $\rho$-labeling.

Now we analyze the injectivity of the function $f$. When $f$ is restricted to the vertices in $T$ it is injective because it is a graceful labeling. Each extension of $f$ to the vertices $u_{i}$ and $w_{i}$ is defined as a linear function, which implies that we just need to check that all the labels used are different.

Note that $\min \left\{f\left(u_{i}\right): 1 \leq i \leq k\right\}=f\left(v_{k}\right)+n+3 k+1$ and $\max \left\{f\left(u_{i}\right): 1 \leq i \leq k\right\}=f\left(v_{1}\right)+n+4 k$. Since $f\left(v_{k}\right), f\left(v_{1}\right) \in\{0,1, \ldots, n\}$, we get

$$
\left\{f\left(u_{i}\right): 1 \leq i \leq k\right\} \subseteq\{n+3 k+1, n+3 k+2, \ldots, 2 n+4 k\}
$$

and

$$
\{0,1, \ldots, n\} \cap\left\{f\left(u_{i}\right): 1 \leq i \leq k\right\}=\varnothing .
$$

On the other side, $\min \left\{f\left(w_{i}\right): 1 \leq i \leq k\right\}=f\left(v_{k}\right)+k+1$ and $\max \left\{f\left(w_{i}\right): 1 \leq i \leq k\right\}=f\left(v_{1}\right)+3 k-1$. Thus,

$$
\left\{f\left(w_{i}\right): 1 \leq i \leq k\right\} \cap\left\{f\left(u_{i}\right): 1 \leq i \leq k\right\}=\varnothing
$$

and

$$
\{0,1, \ldots, n\} \cap\left\{f\left(w_{i}\right): 1 \leq i \leq k\right\}=\varnothing
$$

when $\min \left\{f\left(w_{i}\right): 1 \leq i \leq k\right\}>n$, that is, when $f\left(v_{k}\right)+k+1>n$; which is equivalent to say that $f\left(v_{k}\right)>n-k-1$ or $f\left(v_{k}\right) \geq n-k$. But this condition is one of the hypotheses. Therefore, the final labeling of $T^{\prime}$ is in fact a $\rho$-labeling as we claimed.

Suppose that $T^{\prime}$ has been labeled using the scheme presented above. Then the edges $v_{i} u_{i}$ have weights $n+4 k+1-i$ for every $1 \leq i \leq k$. The edges $u_{i} w_{i}$ have weights $n+2 k+1-i$; that is, the weights $n+k+1, n+k+2, \ldots, n+2 k$.

Recall that if $v_{1}, v_{2}, \ldots, v_{k}$ are the selected vertices of $T$, then $f\left(v_{1}\right)>f\left(v_{2}\right)>\cdots>f\left(v_{k}\right)$. Thus, the edge $u_{k} v_{k}$ has weight $n+3 k+1$, which implies that $f\left(u_{k}\right)=f\left(v_{k}\right)+n+3 k+1$. The edge $u_{k} w_{k}$ has weight $n+k+1$ and $f\left(w_{k}\right)=f\left(u_{k}\right)-n-k-1$, then

$$
f\left(w_{k}\right)=f\left(v_{k}\right)+n+3 k+1-n-k-1=f\left(v_{k}\right)+2 k .
$$

But $f\left(w_{k}\right)$ is the smallest label assigned to a vertex $w_{i}$, then it must be larger than $n$, that is, $f\left(v_{k}\right)+2 k \geq n+1$, in other terms, $f\left(v_{k}\right) \geq$ $n+1-2 k$.

This implies that if the smallest label of the selected vertices of $T$ is at least $n+1-2 k$, the tree $T^{\prime}$ is a $\rho$-tree. Since 0 is the smallest possible value for $f\left(v_{k}\right)$, we get that when $2 k \geq n+1$, any possible tree $T^{\prime}$, obtained from $T$ by attaching $k$ copies of $P_{3}$, is a $\rho$-tree. In this way, we have proven the next theorem. 
Theorem 2.12. If $T^{\prime}$ is obtained from a graceful tree $T$ of size $n$ attaching a copy of $P_{3}$ to each of $k$ selected vertices of $T$ and $2 k \geq n+1$, then $T^{\prime}$ is a $\rho$-tree.

Even when this result is not as strong as Theorem 2.1, it produces $\rho$-labelings for many trees. For instance, if $n=10$, then $6 \leq k \leq 11$; this means that for any graceful tree of size 10 , it is possible to construct $\sum_{k=6}^{11} C(10, k)=2^{10} \rho$-labeled trees. In general we have that there are $\sum_{\left\lceil\frac{n+1}{2}\right\rceil}^{n+1} C(n, k)=2^{n}$ obtained from a gracefully labeled tree of size $n$. In Figure 2.2 we have an example for each $k \in\{6,7,8,9,10,11\}$, where $T$ is a gracefully labeled tree of size $n=10$.
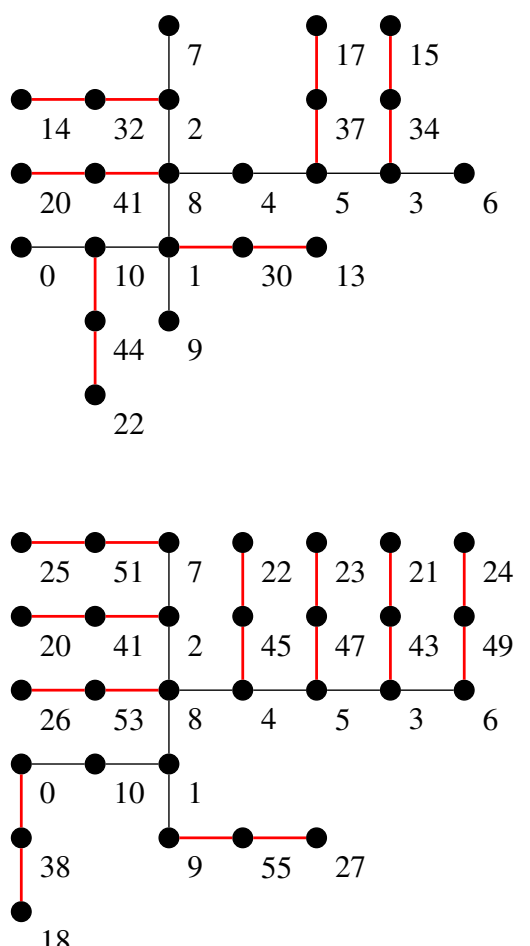

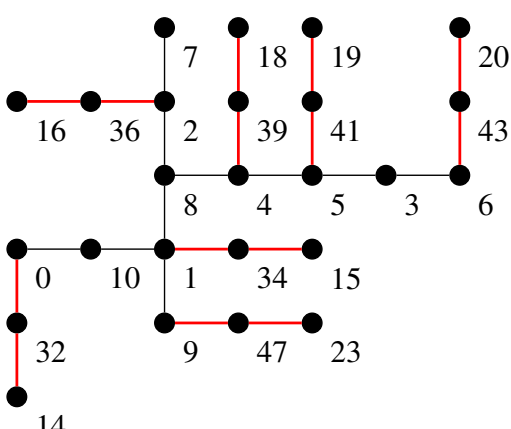

14

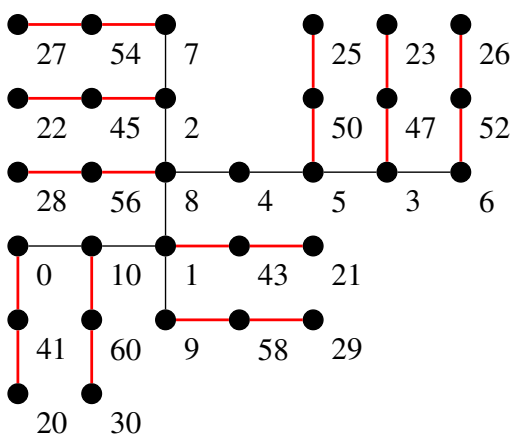

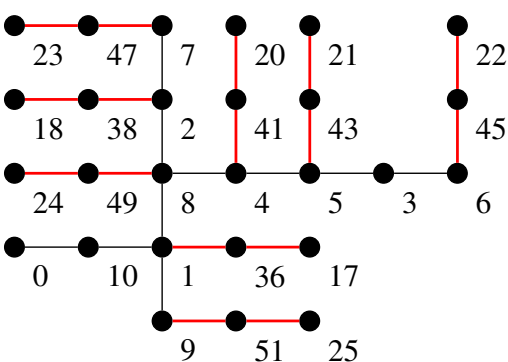

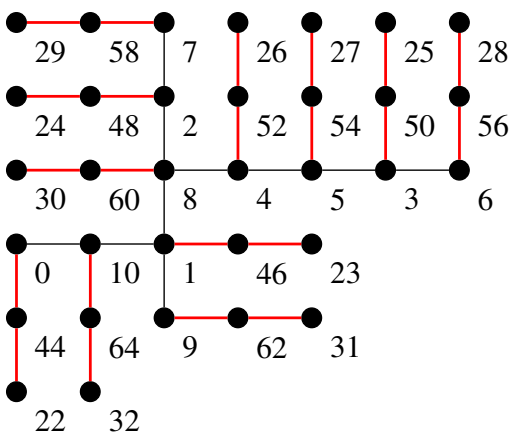

Figure 2.2: Some of the $2^{10} \rho$-labeled trees obtained from a graceful tree of size 10

By $\mathscr{T}_{n}$ we denote the family of all $\rho$-labeled trees which $\rho$-labeling is obtained following the labeling scheme used in Theorem 2.1 .

Theorem 2.13. If $T^{\prime \prime}$ is obtained from $T^{\prime}$ in $\mathscr{T}_{n}$ by attaching a pendant vertex to each of $k$ selected vertices of $T^{\prime}$, where $2 k \geq n+1$ and the labels of any pair of selected vertices are at least two units apart, then $T^{\prime \prime}$ is a $\rho$-tree.

Proof. Assume that $T$ is a graceful tree of size $n$ and $T^{\prime}$ is any tree obtained by attaching any number of pendant vertices to any number of vertices of $T$. Suppose that $t$ is the total number of pendant vertices attached to $T$ to form $T^{\prime}$. Let $f$ be the $\rho$-labeling of $T^{\prime}$ procured using Theorem 2.1 and $v_{1}, v_{2}, \ldots, v_{k}$ be distinct pendant vertices of $T^{\prime}$ such that for each $1 \leq i \leq k, f\left(v_{i}\right)-f\left(v_{i+1}\right) \geq 2$. Note that the labels assigned to the pendant vertices introduced to form $T^{\prime}$ are in the set $\{n+t+2 k+1, n+t+2 k+2, \ldots, n+2 t+2 k\}$. Thus, the integers in the set $L=\{n+1, n+2, \ldots, n+t+2 k\}$ have not been used as labels of $T^{\prime}$.

As we did in the previous theorem, the goal is that the edges generated connecting each $v_{i}$, for $1 \leq i \leq k$, to a pendant vertex, have weights $n+t+k, n+t+k-1, \ldots, n+t+1$, respectively. To achieve this goal, we label the pendant vertices with integers from $L$. Note that $f\left(v_{k}\right) \leq n+t+2 k+1$ and the pendant edge associated with $v_{k}$ has weight $n+t+1$, so the pendant vertex has a label at least $x$, where

$$
\begin{aligned}
(n+2 t+2 k)-x & =n+t+k+1 \\
x & =2 k,
\end{aligned}
$$

since $2 k \geq n+1$, this integer has not been assigned as a label, hence it can be used now and no repetition of labels happens. Similarly, the pendant edge associated with $v_{1}$ has weight $n+t+k$, so the pendant vertex has a label at most $y$, where

$$
\begin{aligned}
(n+2 t+2 k)-y & =n+t+k+1 \\
y & =t+k-1 .
\end{aligned}
$$

Thus, we conclude that is possible to label all the pendant vertices with integers from $L$, producing the weights $n+t+1, n+t+2, \ldots, n+t+k$. Consequently, the labeling of $T^{\prime \prime}$ is in fact a $\rho$-labeling.

In Figure 2.3 we present an example of this labeling for a graceful tree of size 6, with $t=21$ and $k=9$. The blue edges are those produced by the first expansion, and the red edges are the ones created by the second expansion of the graceful tree represented with black edges.a 


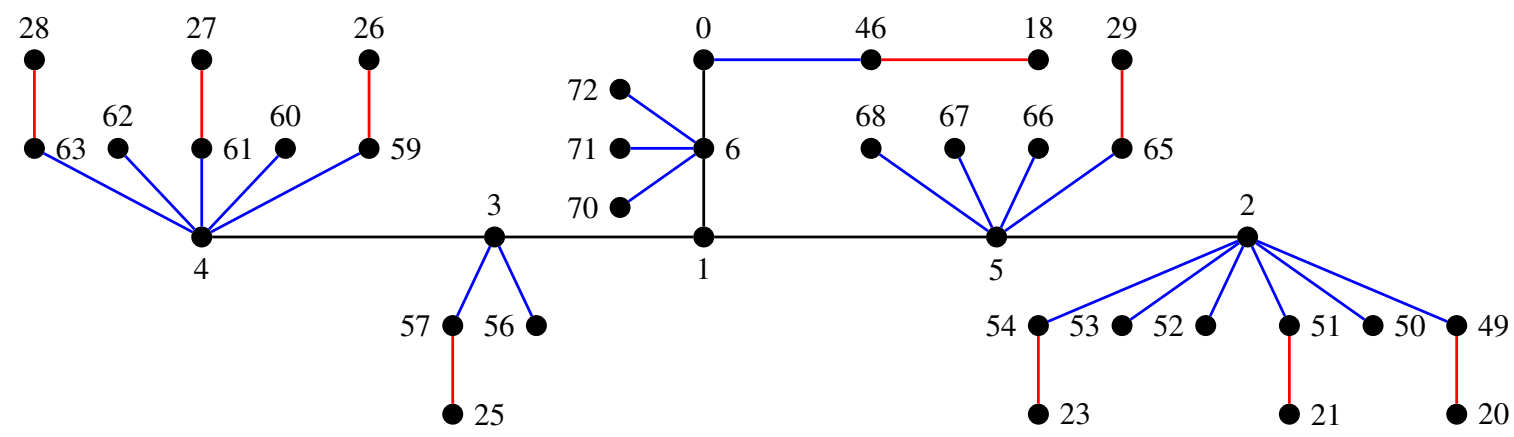

Figure 2.3: A $\rho$-labeled tree obtained from a subdivision of the star $S_{3}$

\section{Every tree is a spanning tree of an $\alpha$-graph}

In this section we show that any tree $T$ of size $n$ is a spanning tree of an $\alpha$-graph of size $n+\varepsilon(T)$, where $\varepsilon(T)$ is the excess of $T$, that is, a parameter associated with the left-layered representation of $T$ introduced in [4].

Let $T$ be a rooted tree, for every vertex $v$ of $T, \gamma(v)$ is the number of levels in $T$ where $v$ has at least one descendant. We say that $T$ is a left-layered tree if the vertices on each level of $T$ adhere to the following rules:

(i) If $u$ and $v$ are siblings of degree one, the order of $u$ and $v$ is arbitrary.

(ii) If $u$ and $v$ are siblings and $\gamma(u)<\gamma(v)$, then $u$ is placed to the left of $v$.

(iii) If $u$ and $v$ are siblings, $\gamma(u)=\gamma(v)$, and $\operatorname{deg}(u) \geq \operatorname{deg}(v)$, then $u$ is placed to the left of $v$.

(iv) If $u$ and $v$ are siblings such that $u$ is placed to the left of $v$, then the descendants of $u$ are placed to the left of the descendants of $v$.

In Figure 3.1 we show the left-layered representation of a tree of size 58. The edges of this tree are represented with black lines.

Let $T_{r}$ be the left-layered representation of a rooted tree $T$ with root $r$. Thus, $L_{k}=\left\{v_{j}^{k}: 1 \leq j \leq n_{k}\right\}$ is the set of all vertices of $T$ at distance $k$ from $r$, that is, the vertices of $T_{r}$ placed on level $k$. We assume that $v_{j}^{k}$ is placed to the left of $v_{j+1}^{k}$, for all $1 \leq j \leq n_{k}-1$. The excess of $L_{k}$, denoted $\Omega_{k}$, is defined to be

$$
\Omega_{k}= \begin{cases}0 & \text { if } k=0, h, \\ n_{k}-n_{k, 0}-1 & \text { if } 1 \leq k \leq h-1,\end{cases}
$$

where $n_{k, 0}$ is the number of vertices in $L_{k}$ with no children and $h$ is the height of $T_{r}$. The excess of $T_{r}$, denoted by $\operatorname{ex}\left(T_{r}\right)$, is given by $\operatorname{ex}\left(T_{r}\right)=\sum_{k=0}^{h} \Omega_{k}$. The excess of $T$, denoted by $\varepsilon(T)$, is defined to be

$$
\varepsilon(T)=\min \left\{\operatorname{ex}\left(T_{r}\right): \text { for all } r \in V(T)\right\} .
$$

Thus, if $T$ is a caterpillar or a path, $\varepsilon(T)=0$, and it is obtained when $r$ is chosen to be any of the vertices of maximum eccentricity in $T$. The tree in Figure 3.1, represented with the black edges, has excess 18; the vertices used to calculate this parameter are in black.

A bipartite labeling of a tree $T$ of size $n$ is an injective mapping $f: V(G) \rightarrow\{0,1, \ldots, s\}$, with $s \geq n$, such that all induced weights are distinct and the labels assigned to the vertices in one of the stable sets of $T$ are smaller than the labels assigned to the vertices of the other stable set. When $s=n$, the bipartite labeling is indeed an $\alpha$-labeling of $T$. In [5] we introduced a bipartite labeling of $T$ where $s=n+\varepsilon(T)$. For the sake of completeness, we describe this labeling again. We will use it to prove that every tree $T$ of size $n$ and excess $\varepsilon(T)$ is a spanning tree of an $\alpha$-graph of order $n+1$ and size $n+\varepsilon(T)$.

Recall that on every level, the vertices are ordered from the right to the left. If $h$ is the height of $T$, then the labels assigned to the vertices on each level are consecutive integers. On the levels $L_{h}, L_{h-2}, \ldots$ they are in increasing order; on the levels $L_{h-1}, L_{h-3}, \ldots$ they are in decreasing order. The smaller labels are on the levels $L_{h}, L_{h-2}, \ldots$.

The first label on $L_{h}$ is 0 and the first label on $L_{h-2 i}$ is the addition of the largest label on $L_{h-2 i+2}$ and $1+\Omega_{h-2 i+1}$.

The first label on $L_{h-1}$ is $n+\varepsilon(T)$ and the first label on $L_{h-1-2 i}$ is the subtraction of the smallest label on $L_{h+1-2 i}$ and $1+\Omega_{h-2 i}$.

In Figure 3.1 we exhibit an example of this bipartite labeling.

Theorem 3.1. Any tree $T$ of size $n$ is a spanning tree of an $\alpha$-graph of size $n+\varepsilon(T)$.

Proof. Suppose that $T$ has been labeled using the bipartite labeling $f$ described before. Thus, the labels assigned belong to the set $\{0,1, \ldots, n+\varepsilon(T)\}$ and the induced weights are in the set $\{1,2, \ldots, n+\varepsilon(T)\}$. Let $v_{1}$ and $v_{2}$ be two consecutive vertices on level $L_{k}$ such that they are not siblings, this implies the existence of two consecutive vertices, $u_{1}$ and $u_{2}$, on level $L_{k-1}$ such that $u_{1} v_{1}, u_{2} v_{2} \in E(T)$.

If $h$ and $k$ have the same parity, then $f\left(v_{1}\right)+1=f\left(v_{2}\right)$ and $f\left(u_{1}\right)-1=f\left(u_{2}\right)$. Thus, the weight of $u_{1} v_{1}$, is $f\left(u_{1}\right)-f\left(v_{1}\right)$, which is two units larger than the weight of $u_{2} v_{2}$, because

$$
f\left(u_{2}\right)-f\left(v_{2}\right)=f\left(u_{1}\right)-1-\left(f\left(v_{1}\right)+1\right)=f\left(u_{1}\right)-f\left(v_{1}\right)-2 .
$$




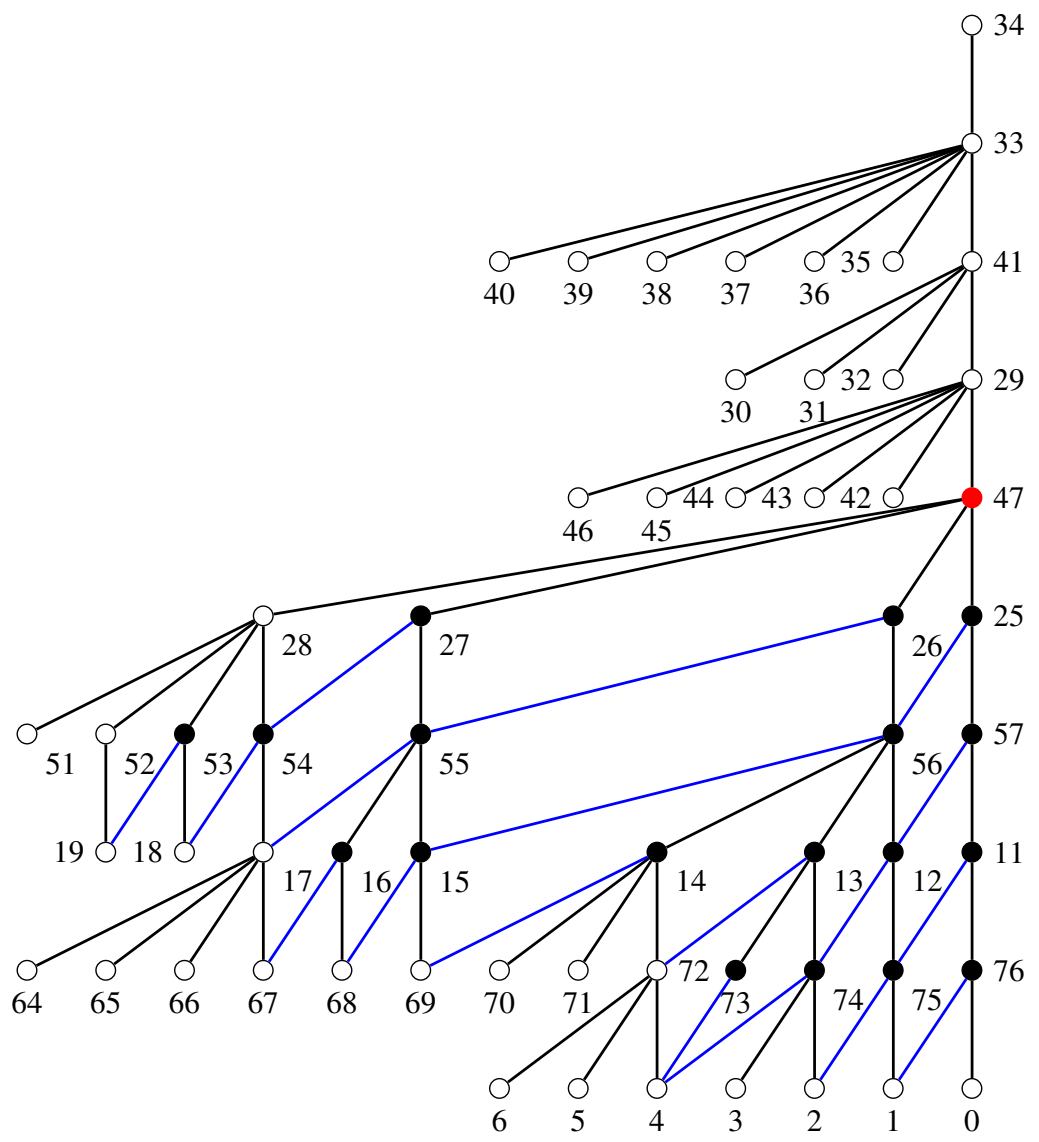

Figure 3.1: Embedding of a tree of size 58 and excess 18 in an $\alpha$-graph of size 76

Hence, the integer $f\left(u_{1}\right)-f\left(v_{1}\right)-1$ is not the weight of any edge of $T$. If the vertices $v_{2}$ and $u_{1}$ are connected (or the vertices $v_{1}$ and $u_{2}$ ), we create an edge of weight $f\left(u_{1}\right)-f\left(v_{1}\right)-1$.

If $h$ and $k$ have different parity, then $f\left(v_{2}\right)=f\left(v_{1}\right)-1$ and $f\left(u_{1}\right)+1=f\left(u_{2}\right)$. Thus, the weight of $u_{1} v_{1}$, is $f\left(u_{1}\right)-f\left(v_{1}\right)$, and again, it is two units larger than the weight of $u_{2} v_{2}$, because

$$
f\left(v_{2}\right)-f\left(u_{2}\right)=f\left(v_{1}\right)-1-\left(f\left(u_{1}\right)+1\right)=f\left(v_{1}\right)-f\left(u_{1}\right)-2 .
$$

So, the integer $f\left(v_{1}\right)-f\left(u_{1}\right)-1$ is not the weight of any edge of $T$. If the vertices $v_{2}$ and $u_{1}$ are connected (or the vertices $v_{1}$ and $u_{2}$ ), we create an edge of weight $f\left(v_{1}\right)-f\left(u_{1}\right)-1$.

If this process of introducing a new edge is done every time that is needed, the resulting graph has $T$ as a spanning tree and its labeling induces the weights $1,2, \ldots, n+\varepsilon(T)$. Since the original labeling is bipartite, the labeling of the new graph is an $\alpha$-labeling.

In Figure 3.1 we show an example of this labeling. The black edges correspond to a tree $T$ of size $58, \varepsilon(T)=18$, and $h=9, \Omega_{9}=\Omega_{4}=$ $\Omega_{3}=\Omega_{2}=\Omega_{1}=\Omega_{0}=0, \Omega_{8}=4, \Omega_{7}=6, \Omega_{6}=5$, and $\Omega_{5}=3$. The missing weights are 27, 29, 31, 34, 36, 38, 41, 45, 51, 53, 59, 62, $69,70,73$, and 75 . If the blue edges are added to the labeled tree, they form an $\alpha$-labeled graph of size $58+18=76$ that contains $T$ as a spanning tree.

Note that for every missing weight in the original bipartite labeling of $T$, there are two possible new edges with that weight, therefore, there are at least $2^{\varepsilon(T)}$ hosts for $T$ that are $\alpha$-graphs. As far as we know, the $\alpha$-graph obtained in this way, is the smallest $\alpha$-graph that has $T$ as a spanning tree.

Other authors have studied similar problems; for example, Rao and Sahoo [24] proved that every connected graph of order $n$ can be embedded as an induced subgraph in a graceful Eulerian graph of size $3^{n}$. In [25], Sethuraman and Ragukumar introduced some algorithms that allow them to prove, among other results, that any tree of size $n$ is a spanning tree of an $\alpha$-graph of size $M$. They provided an example for a tree of size 20 embedded in an $\alpha$-graph of size 55. This tree has excess 5; thus, when we apply our labeling scheme to this tree, the associated $\alpha$-graph has size 25. We have not been able to determine the value of $M$ in [25] but we believe that for any given tree $T$ of size $n$, the inequality $n+\varepsilon(T)<M$ holds.

\section{Conclusion}

Since there are many known families of graceful trees and any graceful tree can be used to construct infinitely many larger $\rho$-trees, Theorem 2.1 is the most powerful result in the context of $\rho$-labelings. Then it was natural to ask whether is possible to weaken the condition of being graceful imposed to the starting tree. As a result of that analysis, we obtained the last theorems in Section 2. In particular, Theorem 2.13 tells us that for any $k$ large enough, there are $k$ end-vertices in $T^{\prime}$ that can be extended one more time, to obtain a new $\rho$-tree. Is there any other 
type of tree that can be used, instead of $P_{3}$, to attach to all or some of the vertices of a tree $T$ to create new $\rho$-trees? The result in Section 3 gives us an upper bound for the size of the smaller $\alpha$-graph that contains $T$ as a spanning tree. Further studies of this type should include the examination of how good this bound is, which is better than any other bound known to date.

\section{Acknowledgement}

We want to thank Dr. David Williams for helping us to find part of the bibliography used here.

\section{References}

[1] G. Ringel, Problem 25, in Theory of Graphs and Its Applications, Proc. Symposium Smolenice 1963, Prague (1964), 162.

[2] A. Kotzig, On certain vertex valuations of finite graphs, Util. Math., 4 (1973), 67-73.

[3] A. Rosa, On certain valuations of the vertices of a graph, Theory of Graphs (Internat. Symposium, Rome, July 1966), Gordon and Breach, N. Y. and Dunod Paris (1967), 349-355.

[4] C. Barrientos, E. Krop, Improved bounds for relaxed graceful trees, Graphs Combin., 33 (2017), $287-305$.

[5] C. Barrientos, S. Minion, New attack on Kotzig's conjecture, Electron. J. Graph Theory Appl., 4(2) (2016), $119-131$.

[6] G. Chartrand, L. Lesniak, Graphs \& Digraphs, 2nd ed. Wadsworth \& Brooks/Cole, Monterey, 1986.

[7] J. A. Gallian, A dynamic survey of graph labeling, Electronic J. Combin., 21(\#DS6), 2018.

[8] Y. Caro, Y. Roditty, J. Schönheim, Starters for symmetric $(n, G, 1)$-designs. $\rho$-labelings revisited, (in press).

[9] A. Kézdy, $\rho$-valuations for some stunted trees, Discrete Math., 306 (2006), 2786- 2789.

[10] P. Bahl, S. Lake, A. Wertheim, Gracefulness of families of spiders, Involve, 3 (2010), 241-247.

[11] S. El-Zanati, C. Vanden Eynden, N. Punnim, On the cyclic decomposition of complete graphs into bipartite graphs, Australas. J. Combin., 24 (2001), 209-219.

[12] C. Huang, A. Rosa, Decomposition of complete graphs into trees, Ars Combin., 5 (1978), $23-63$.

[13] D. Morgan, All lobsters with perfect matchings are graceful, Electron. Notes Discrete Math., 11 (2002), 6 pp.

[14] M. Burzio, G. Ferrarese, The subdivision graph of a graceful tree is a graceful tree, Discrete Math., 181 (1998), $275-281$.

[15] W. Fang, A computational approach to the graceful tree conjecture, arXiv:1003.3045v1 [cs.DM].

[16] K. Eshghi, P. Azimi, Applications of mathematical programming in graceful labeling of graphs, J. Applied Math., 1 (2004), 1-8.

[17] C. Huang, A. Kotzig, A. Rosa, Further results on tree labellings, Util. Math., 21c (1982), 31-48.

[18] S. K. Vaidya, N. A. Dani, Cordial labeling and arbitrary super subdivision of some graphs, Inter. J. Information Sci. Comput. Math., 2(1) (2010), 51-60.

[19] D. J. Jin, F. H. Meng, J. G. Wang, The gracefulness of trees with diameter 4, Acta Sci. Natur. Univ. Jilin., (1993), 17-22.

[20] P. Hrnčiar, A. Haviar, All trees of diameter five are graceful, Discrete Math., 233 (2001), 133-150.

[21] J. C. Bermond, D. Sotteau, Graph decompositions and G-design, Proceedings of the Fifth British Combinatorial Conference, 1975, Congr. Numer., XV (1976) 53-72

[22] C. Balbuena, P. García-Vázquez, X. Marcote, J. C. Valenzuela, Trees having an even or quasi even degree sequence are graceful, Applied Math. Letters, 20 (2007), 370-375

[23] G. Sethuraman, J. Jesintha, A new class of graceful rooted trees, J. Disc. Math. Sci. Crypt., 11 (2008), $421-435$.

[24] S. B. Rao, U. K. Sahoo, Embeddings in Eulerian graceful graphs, Australasian J. Comb., 62(1) (2015), 128-139.

[25] G. Sethuraman, P. Ragukumar, Every tree is a subtree of graceful tree, graceful graph and alpha-labeled graph, Ars Combin., 132 (2017), 105-109. 